Velocity of ultrasonics in liquids by an optical method. A form of optical stroboscope has been devised, using two sets of ultrasonic progressive waves passing through a transparent liquid in a small tank, from opposite directions, parallel but one behind the other. If a light beam crosses the glass tank at right angles to the two beams, it will be 'chopped' at double the frequency of the two sets of progressive waves. The light so interrupted can be used to illuminate another small tank containing the liquid under examination, which is itself irradiated at double the frequency of the primary sources by means of electrical coupling between this third quartz and the other two. In the light which passes the latter tank are seen dark bands at a separation equal to the ultra. sonic wave-length in the experimental liquid. These spacings are measured by a microscope, and so the velocity in the liquid derived (Fig. 2).

Attenuation of ultrasonics in liquids. This apparatus likewise consists of an irradiated liquid crossed by a parallel beam of light from a mercury are, the location of which along the ultrasonic beam can be varied. At each station, the intensity of the first-order diffracted light is measured with a photo-electric cell. It has been shown that the latter is directly proportional to the ultrasonic intensity at the place in question if the quartz source is not overdriven. From a set of such readings the attenuation along the ultrasonic beam is deduced. It has been found very necessary to watch the constancy of both light and sound sources if accurate results are to be obtained. One is assured by setting a photo-electric cell near the mercury arc, and the other by driving the quartz through a battery-operated dynamo-converter.

Properties of ultrasonic lenses made of 'Plexiglass'. The lenses are plano-concave, mounted at a distance from the quartz emitter or directly attached to it. The efficiency and properties of such lenses are being studied as a means of increasing the energy density locally of ultrasonics in a liquid.

Biological applications of ultrasonics. Oscillators of $800 \mathrm{kc} / \mathrm{sec} ., 2 \mathrm{Mic} / \mathrm{sec}$. and $4 \mathrm{Mc} / \mathrm{sec}$. are used, immersed in a liquid carefully chosen to allow the maximum of intensity in the ultrasonic beam. This passes through a thin window-also chosen for its material and thickness so as to give maximum transmission-into the test tank which contains the bacterial culture. Experiments of this nature have, of course, been performed before, but many of them without proper control of ultrasonic intensity. In this work the temperature of the liquid containing the culture is carefully measured, as the bacteria are very sensitive to heat, while the holder of the quartz source is so designed that it is possible to calculate the intensity emitted into the liquid from its form and dimensions and from those of the windows and liquid media used.

The National Council for Research itself is an organisation which supports, and to a certain extent directs, scientific research in Italy out of funds provided by the Italian Government. The Administration Building (Fig. 1) opposite the University of Rome also houses the three Institutes directly under the control of the National Council, which is responsible for the salaries of their personnel. Besides the Institute for Ultra-acoustics, there is an Institute for the Applications of Calculus and the Institute for Nutritional Research. These three are in close relationship to the Science Faculty of the University of Rome. There is a library which, as far as scientific periodicals are concerned, is the best library in Italy. In recent years, this library has been helped by the British Council to make good some of its losses and gaps in British periodicals occasioned by the War. It admits accredited outside readers, for example, research students and fellows of universities, and maintains a micro-film service.

In addition to these maintained Institutes, the Council administers grants-in-aid to about seventy centres of scientific research within the Italian universities, providing funds for apparatus and postgraduate maintenance. Its scientific activities are governed by a number of committees of university professors under the general supervision of its president (Prof. G. Colonnetti) and secretary-general (Dr. A. Morelli). The organisation is, nevertheless, woefully lacking in funds and deserves more financial support from the Italian Government.

E. G. RICHARDSON

${ }^{1}$ J. Acoust. Soc. Amer., 20, 328 (1948).

\section{ANNUAL CONFERENCE OF THE TEXTILE INSTITUTE}

$T$ HE Annugleonference of the Textile Institute, which w $\$$ held during May 24-27 in Dublin, met to discrys "The Industrial Application of New Knowledges. In an introductory paper, the chairman of the Conference Committee made cled Institute's intention to discuss, not reseangn as an activity, but the ways in which the textil findustry transmitted and utilized new know. ledge Hewed as the end-product of research activity.

Nineteen papers were presented, made up of contributions from research associations, including the National Physical Laboratory, industrial research laboratories and development units, mill managements and a university department. It is in the nature of the textile industry, which is, in fact, many industries, that something of a miscellany should be provided by the titles. Thus an example of physical research immediately assimilable by the industry was provided in a paper on the acoustics of sound reduction; and the application of new knowledge to textile performance standards was illustrated by a paper on dimensional changes in socks. For all the six full sessions of the Conference, it was inevitable that the papers should, as indicated by these examples, spread somewhat thinly over a wide range of topics.

A pattern did, however, emerge, and the following general headings provide the outline: (a) lines of communication between sources of new knowledge and the factory ; $(b)$ winning of new knowledge from practical mill experimentation; (c) utilization of new knowledge in controlling the production of, and standardizing the performance of, consumer goods; (d) utilization of research findings from sources outside the textile industry ; $(e)$ transmission to other industries of new knowledge gained in the study of textile materials ; $(f)$ formal education of scientific workers required to fit them for research and its utilization.

This group of topics served to bring together directors, managers, technicians and research workers in discussion, such being the prime intention of the Conference organisers. There were collisions. A frequently recurring item of dispute concerned the difficulty experienced by management in understanding reports and papers written by scientific workers. The latter were accused of being needlessly obscure. 
This discussion, which could have been foreseen, was surprisingly fruitful. It served to pin-point a vital weakness which now exists throughout the industry, namely, that in undertakings which, in a greater or lesser degree of urgency, require to assimilate new knowledge, there exists all too rarely the scientific man-power capable of applying it rapidly to existing techniques. Mill management cannot be expected to understand the language of the chemist, the physicist and the statistician. But if the output of research institutions is to be assimilated, there must be more widely employed the kind of worker who can understand the language of science and make constructive proposals for its translation into action, and also can assist mill management in formulating the right questions to ask of research. This latter function came out as an essential need; almost all textile operations are complex to a degree which calls for deep technical and scientific insight if questions about them are to be clearly formulated. All too often the wrong questions are asked.

The method of transmission of new ideas, from any source, to spinning room, weaving shed and dyehouse is the core of the problem which was before the Conference. Three papers dealt directly with this. J. H. Black gave a detailed account of the way in which the British Cotton Industry Research Association is organised for transmission. He brought out the essential functions of a research association, showed its inner workings in a historical account of the Shirley Institute, and taught that the first of these functions was not research, but education. The more important work began after new knowledge had been acquired by the Institute. He estimated that about fifty per cent of the industry which subscribed to investigations supported by the Department of Scientific and Industrial Research were receiving what new knowledge was transmitted. This section of the industry applied transmitted ideas more or less directly; the rest received them slowly by a process of permeation. Mr. G. R. Perdue described the information service available to members of the British Launderers Research Association in similar terms. Mr. W. Howarth, a representative of mill managements, dealt with the handling of operatives, viewing any change in established mill technique as a human experience rather than as a mere mechanical point of departure. He made the interesting observation that 'intuitive' female workers were more receptive to new technical departures than the more 'logical' male operatives as a general rule.

Discussion of practical mill experiments touched on two major difficulties. In the small undertaking sufficient data under any given set of working conditions can rarely be amassed for useful statistical treatment. In the large undertaking, on the other hand, it is rarely possible to amass such data without expensive interferences with production. Many acute problems, especially in sizing, spinning and weaving, can only be approached by the statistical treatment of exhaustive observations. Further, in the institution of new techniques, there is often no pilot-plant stage ; the card-room or the weaving shed must be its own pilot plant. Papers by E. Bradbury and $\mathrm{H}$. Hacking, dealing with sizing and weaving, and by J. Ingham on worsted yarn manufacture, and the discussions which followed them revealed the dilemmas implicit in the above, and pointed to the importance of full co-operation of member firms in providing research associations with facilities for the highly co-ordinated field-work which mill experimentation calls for.
A lengthy and detailed account by R. A. McFarlane and C. C. Wilcock of the controls employed in the dyeing and finishing of rayon piece goods provided an example of a dyehouse management and a research department, in the same large organisation, working hand-in-glove to ensure efficient production to a predetermined standard of quality by integration, on the plant, of research findings with traditional practice. Other papers on control were: assessment of yarn quality (M. W. H. Townend), textile material specifications - their value, form and content (G. E. Collins), performance standards for development fabrics (C. P. Tattersfield) and shrinkage in knitted wool fabrics (W. A. Dutton). Discussion of these papers brought out some difficulties peculiar to the industry. To a great degree the textile industry of Great Britain and Ireland is made up of horizontal units. Spinners, weavers and dyers are separate concerns, often dealing with such other separate concerns as twisters, doublers, bleachers, raisers and finishers. In the vertical concern, steps for the control of production standards can be taken in the know. ledge that there will be a corresponding standard of performance in the final product. In horizontally organised industry, a degree of enlightenment and co-operation is called for to attain this same end; but it does not exist. Even if it did, there is the converter. He, having bought a fabric, every element in the production of which may have been carefully controlled, is at perfect liberty to have it made up into garments or hangings for which it was never intended. Will our new knowledge reach out to the merchant converter, and by what means ?

On the subject of new knowledge from outside the textile field, G. S. J. White dealt with textile contributions by the organic chemical industry. These he put forward under (a) polymers, especially fibreforming chain-polymers, (b) colouring matters, (c) surface-active agents.

The dependence of textiles on the last two classes, which cover dyes, pigments, wetting agents, detergents, and a number of products for use in all wet processing, is well understood. The transmission of new knowledge based on organic research and manufacture has accompanied the sale and servicing of the product on one hand and, on the other, has flowed back to the makers from researches carried out by the more enlightened and better equipped of the users. All these are chemical products for use in processing fibres. What of fibres themselves? If these are to be made by the organic chemical industry, the textile industry must co-operate very closely indeed. Only they know what kind of fibres can fill any gaps that may exist in the gamut of natural fibres and rayons; only they can indicate what properties are required to make a usable textile from an organic chain polymer. Mr. White reproduced a table of properties for four synthetic chain polymers, Vinyon $N$ (polymerized acrylonitrile and polyvinyl chloride), Orlon (acrylonitrile), nylon (a super polyamide) and terylene (experimental polyester yarn). These were properties measurable by physical and chemical means, such as colour, density, tenacity and resistance to reagents.

In synthesizing fibres of this type, the organic chemist must be advised by the textile man what combinations of physical and chemical properties are required for textile purposes. The organic chemist can understand and attempt to implement those requirements. But what of textile properties proper, such as drape, resilience, feel, dimensional stability? To integrate these needs into fibre research 
calls for much more than product-servicing contacts between maker and user. The evaluation of any fibre in terms of these properties is a time-consuming process, calling for very close collaboration between all branches of the industry and for some refinement of existing experimental methods. If we understand it correctly, consideration of Mr. White's paper leads to the inevitable conclusion that the textile industry must dictate, in tempo and direction, the textile realization of the products submitted to them by the organic chemist. It is therefore important that, through its own research and development work, the textile industry should be in a position to exert the maximum critical pre-selection of organic chemicals proposed for use as textile fibres.

J. K. Berry described the contributions which rayons, especially high-tenacity regenerated celluloses, are making in engineering fields, and discussions brought out the value of collaboration with, for example, the rubber tyre industry. $\mathrm{He}$ is of the opinion that the expensive high twist required by tyre makers does not necessarily contribute to fatigueresistance, and that there is a possible prejudice about the matter which can only be cleared up by co-operative investigation in both industries.

W. J. Hamburger, director of Fabrics Research Inc., Boston, U.S.A., presented a paper entitled "Industrial Applications of Stress-Strain Relationships". The contents were a sound engineering approach to fine-structure problems. But the author's remarks had to do more with the philosophy of research $\mathrm{He}$ described how a privately owned consulting laboratory not only can afford to have ideals in the prosecution of some fundamental research, but also, in his case, has been able to get industrial clients to pay for it and to discover that it paid them back.

The remaining papers were : the industrial application of sound reduction (N. Fleming) ; solubility number-a measure of cellulose degradation (D. A. Derrett-Smith) ; measuring the swelling of fibres in water (J. M. Preston and M. V. Nimkar); the development of a laundry, washing process (F. C. Harwood and F. R. Hill); University departments their influence on the efficient conduct and utilization of research (H. A. Turner). Messrs. Harwood and Hill revealed how, in the institution of a sound washing technique for laundries, new knowledge was slowly gained and, of necessity, only slowly superimposed on the rule-of-thumb methods of preLaunderers Research Association days. Mr. Turner's paper was the one contribution to formal education of the scientific worker. The Conference was clear on the need for the type of scientific worker who could ensure the transmission of new knowledge to his employers. Mr. Turner considered the way in which he might best be trained, in a university department where technology was properly evaluated and research regarded as a training activity as well as an $e$ d in itself.

The Conference succeeded in revealing many of the obstacles in the way of maximum liaison between research activity and the application of new knowledge. If it were not always made clear how such could be removed, at least the problems involved were clarified, and it now remains for all concerned to contribute towards their solution. In this, through its Transactions, Proceedings and the organisation of specialized conferences, the Textile Institute itself has a unique opportunity of exercising leadership.

JoHN BouLTON

\section{BACTERIAL OXIDATION OF ARSENITE}

\author{
By DR. A. W. TURNER, O.B.E.
}

Scientific and Industrial Research Organisation, Division of Animal Health and Production, Parkville, Victoria

$I^{N}$ 1918 H. H. Green ${ }^{2}$ isolated from arsenical cattledipping fluids in South Africa a bacterium, provisionall referred to as Bacillus arsenoxydans but since/ost ${ }^{2}$, which was able to grow in culture media ch ntaining up to I per cent of $\mathrm{As}_{2} \mathrm{O}_{3}$ as arsenite and to bring about its oxidation to arsenate. In view of the well-known toxicity of tervalent arsenicals, now largely explicable in terms of their inhibitory action upon highly important sulphydryl-dependent metabolic enzymes ${ }^{3,4}$, it is particularly unfortunate that Green's valuable paper, being published in an annual report mainly devoted to veterinary research, apparently escaped the attention of enzymologists and general bacteriologists; for the enzymic mechanism of arsenite oxidation was never studied while the strain was available, nor was the existence of arsenite-oxidizing bacteria ever afterwards reported.

However, during an investigation into spontaneous oxidation of arsenite in cattle-dipping fluids from Queensland, we readily isolated fifteen strains of heterotrophic bacteria which, like Green's bacterium, not only tolerate up to $0 \cdot 1 M$ arsenite, but also bring about its oxidation to arsenate, and we have taken the opportunity to inquire into the mechanism of arsenite oxidation. These bacteria all appear to belong to the genus Pseudomonas; but five distinct types or possibly species can be recognized on the basis of colony characteristics, serological relation. ships, thermal tolerances, pigment production, utilization of carbon sources, and biochemical criteria. One type, found only once, resembles fairly closely the published description of $B$. arsenoxydans.

The enzymic mechanism has been studied mainly with the particularly active strain 17 , belonging to Type 5. In most of the work reported here, arsenite oxidation has been estimated by iodometric titration but in some studies which will be reported later, this has been supplemented and amplified by Warburg respirometry experiments.

Washed cells harvested from suitable liquid media, such as Koser's citrate containing $0.02 M$ sodium arsenite, actively catalyse the oxidation of arsenite to arsenate when aerated. Enzymic activity appears early during growth in arsenite media and is strongly adaptive; non-adapted cells are inactive and develop activity only after a lag-period in the presence of substrate. Activity of washed cells is not dependent upon a source of external phosphate, since it is identical in phosphate or collidine buffers at the same $p \mathrm{H}$. The optimal $p \mathrm{H}$ lies between $6 \cdot 0$ and $6 \cdot 7$, at which arsenite is still almost completely undissociated ( $p K a=9 \cdot 12$ ), and this range is also optimal for growth and arsenite oxidation in arsenite-Koser modium. At the optimal $p \mathrm{H}$, cells aerated optimally can oxidize about $90 \mu M$ arsenite per hour per mgm. dry weight. Oxidation is completely inhibited outside the range $p H$ 3-11. Under the experimental conditions, the optimal temperature for oxidation by aerated cells in phosphate buffer is about $40^{\circ} \mathrm{C}$.; the $Q_{10}$ between $20^{\circ}$ and $30^{\circ}$ is 1.73 , and $Q_{10}$ between $30^{\circ}$ and $40^{\circ}$ is 1.43 ; and between $20^{\circ}$ and $40^{\circ}$ 